\title{
Identification of RET gene fusion by exon array analyses in "pan-negative" lung cancer from never smokers
}

\author{
Cell Research (2012) 22:928-931. doi:10.1038/cr.2012.27; published online 21 February 2012
}

\section{Dear Editor,}

The incidence of lung cancer from never smokers has increased dramatically in China nowadays. Strikingly, approximately $30 \%$ of the lung cancer patients in East Asian population are never smokers $[1,2]$. The majority of these patients are females with lung adenocarcinomas [2]. Identification of oncogenic drivers, which the tumors are "addicted to" and rely on for survival, has significantly reformed the current strategies for lung cancer treatment in clinic and initiated the era of personalized therapy [3]. Therapeutics specifically targeting EGFR mutations, frequently observed in never smoker patients with lung cancer, have been very helpful in improving the clinical symptoms as well as the progression-free survival [4-6]. Similarly, patients with lung tumors positive for $A L K$ fusions also benefit from ALK-targeted therapy $[7,8]$.

Our previous efforts have constructed a quite comprehensive map of those essential oncogenic drivers in 52 lung adenocarcinomas from never smokers [9]. We have uncovered the oncognic drivers in about $90 \%$ of these lung tumors including mutations of EGFR, HER2, KRAS, as well as $E M L 4-A L K$ fusion [9], thus providing a strong clinical guidance for molecular-targeted therapy for this subset of disease. However, there is still about 10\% (5/52) of these never smoker patients were "pan-negative" for all known oncogenic driver mutations and could not benefit from the effective targeted therapy in clinic.

Similar to oncogenic gene mutations, gene fusions such as EML4-ALK or CD74-ROS1 are also essential for lung cancer development $[8,10]$, and serve as effective therapeutic targets. Since great efforts have been paid in searching gene mutations, we instead focus on our efforts in identification of novel oncogenic gene fusions. Previous studies have demonstrated that exon array analyses are capable of detecting gene fusions based on the differential expression of the exons located at either side of the breakpoint, which is frequently resulted from genomic translocation $[7,11,12]$. For example, in the case of $A L K$ fusion, the expression levels of $A L K$ exon
1-20 and exon 21-29 flanking the breakpoint are significantly different and can be readily detected by exon array analyses $[7,11,12]$. Therefore, we performed exon array (Affymetrix Exon 1.0) using all the five "pan-negative" samples plus another 12 samples with known oncogenic drivers to search novel oncogenic gene fusions. We initially identified about 1000 potential gene fusions from exon array analyses. Since most of known oncogenic drivers are kinases, we manually went through all the heatmaps of those potential kinase fusions. Interestingly, we identified one potential RET fusion, with an obvious expressional change between exon 11 and exon 12, in the "pan-negative" lung cancer sample 181LC (Figure 1A). We then preformed the 5' RACE assay to detect the partner of this potential RET fusion. We found that the DNA band obtained from $5^{\prime}$ RACE (about $1.4 \mathrm{~kb}$ ) is actually the fusion of RET exon 12 to CCDC6 exon 1 (Figure $1 \mathrm{~B}-1 \mathrm{C})$, which has been previously reported in human thyroid carcinomas [13]. This RET fusion is derived from somatic genetic alteration since it is undetectable in paired normal lung tissue 181NL (data not shown). To clone the genomic breakpoint, we further designed a series of primers ( 22 forward primers at CCDC6 intron 1 with 1-3 kb intervals, and a reverse primer at RET exon 12) and performed long-range PCR using genomic DNA from the "pan-negative" lung cancer sample 181LC. Interestingly, we found that the intron 1 of CCDC6 is fused to a part of RET exon 11 at genomic DNA level (Figure $1 \mathrm{D}-1 \mathrm{E})$, which results in the expression of CCDC6-RET fusion (CCDC6 exon 1 fused to RET exon 12) after RNA splicing. Previously studies have shown that RET signaling pathway promotes cell survival and cell proliferation through RAS-ERK pathway and PI3K-AKT pathway [14]. RET fusions, mainly found in papillary thyroid carcinomas [15], are oncogenic drivers and capable of transforming thyroid epithelial cells in vitro as well as inducing papillary thyroid carcinoma in transgenic mice $[16,17,18]$. We found that the CCDC6-RET fusion from lung cancer sample $181 \mathrm{LC}$ is undetectable in the rest of 4 "pan-negative" samples as well as those with known oncogenic driver mutations from never smokers (data not 
A

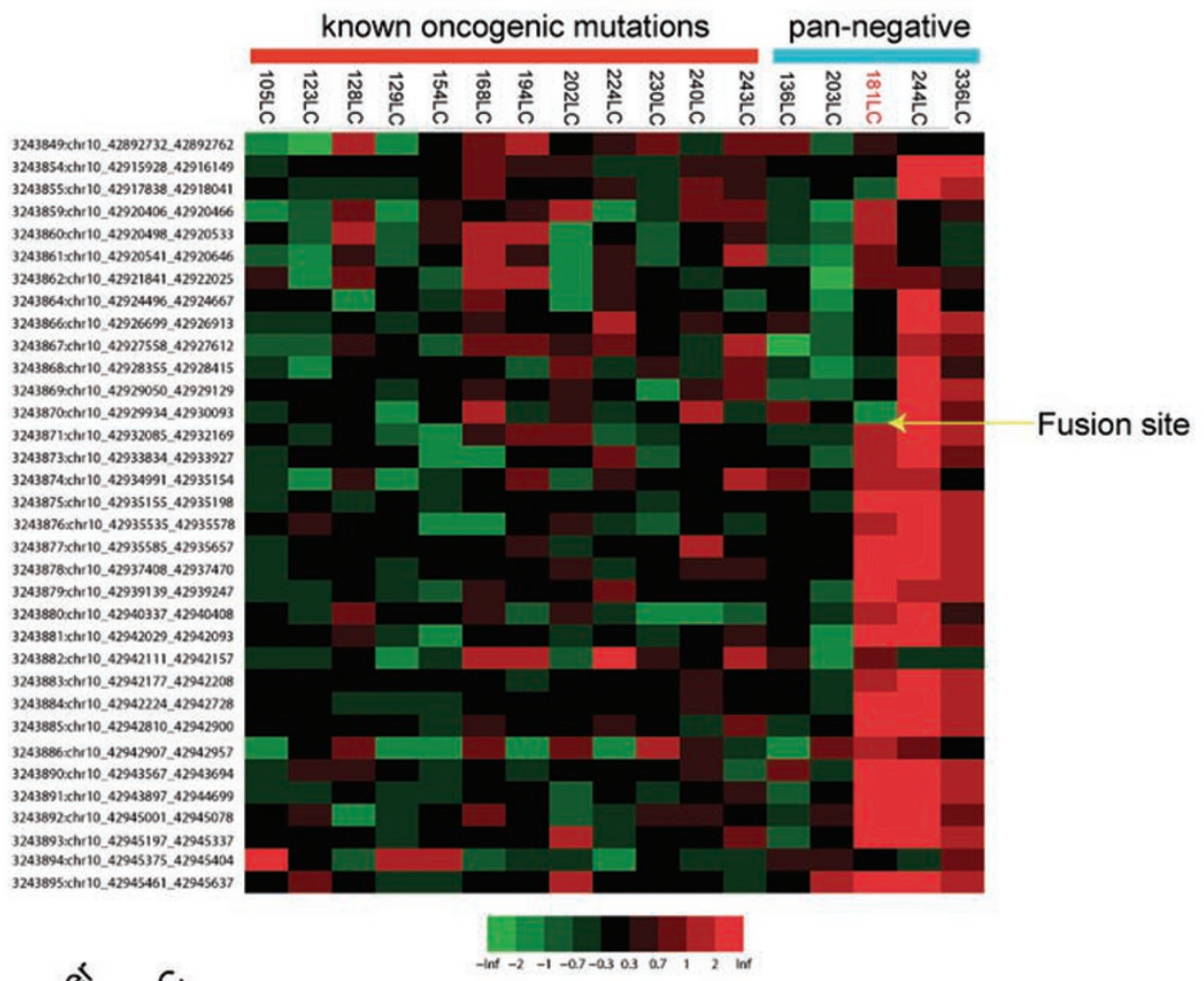

B

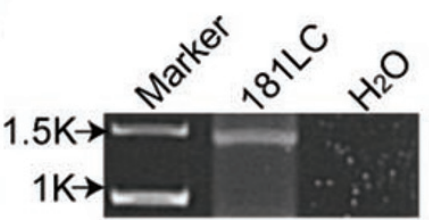

F

C
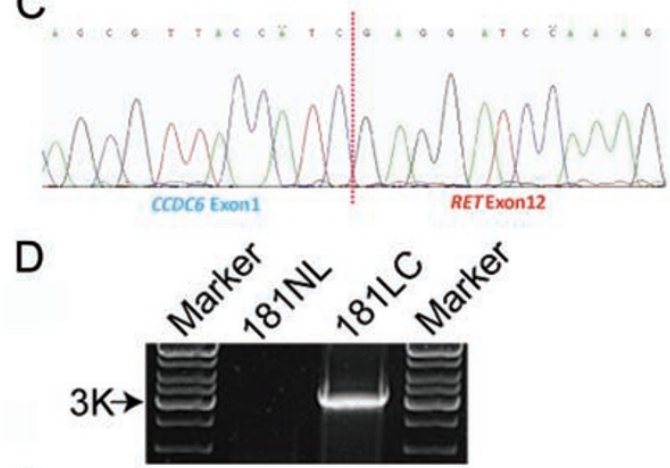

$\mathrm{E}$
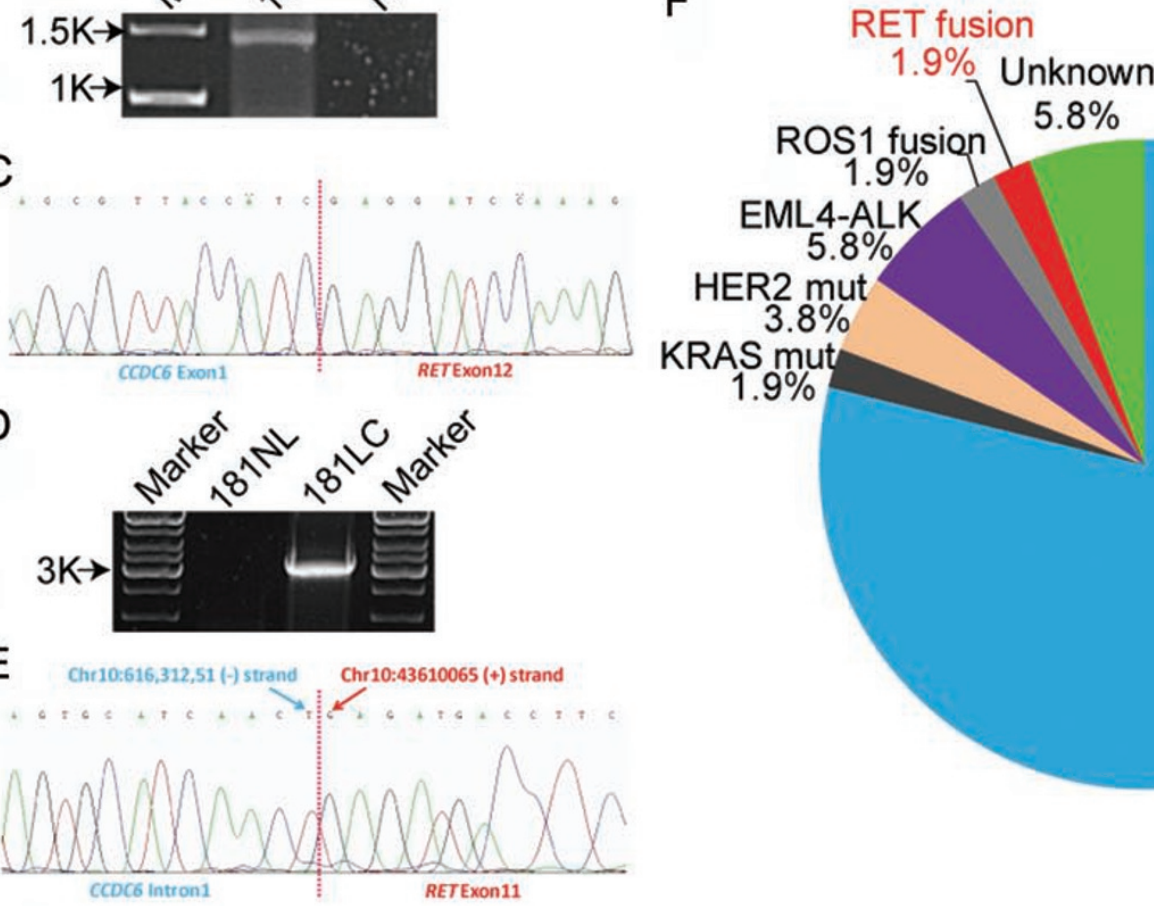

KRAS mut

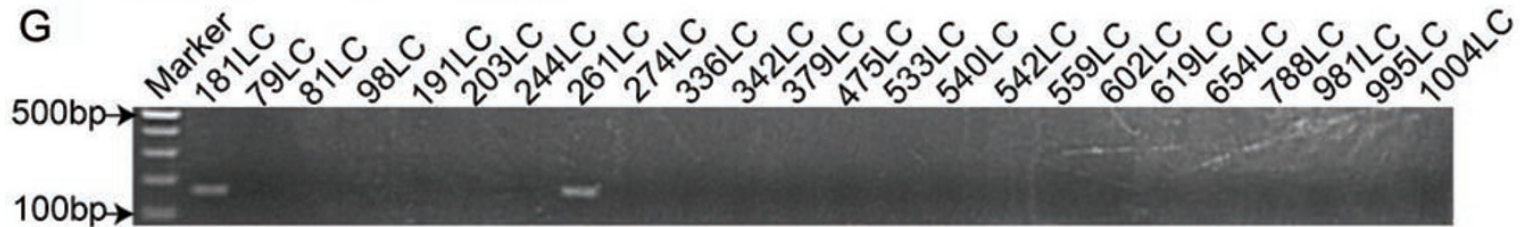


Figure 1 Identification of CCDC6-RET fusion in the "pan-negative" lung adenocarcinomas from never smokers and the construction of a more comprehensive spectrum of oncogenic drivers in this subset of lung cancer. (A) Exon array analyses of 5 samples "pan-negative" for oncogenic drivers and 12 samples with known oncogenic drivers have identified a potential RET fusion in lung cancer sample 181LC. The potential fusion point is indicated by yellow arrow. (B) 5' RACE analyses of the "pan-negative" lung cancer sample 181LC showed a PCR band about $1.4 \mathrm{~kb}$, which is obviously different from the wildtype RET band (about $960 \mathrm{bp}$ ). (C) Sequencing result confirmed the CCDC6-RET fusion in lung cancer sample 181LC. (D) Long-range PCR detection of the genomic breakpoint of CCDC6-RET fusion in lung cancer sample 181LC. (E) Sequencing result showed the detailed genomic breakpoint of CCDC6-RET fusion in lung cancer sample 181LC. (F) A more comprehensive spectrum of oncogenic drivers is constructed for the cohort containing 52 lung adenocarcinomas from never smokers. (G) Detection of CCDC6-RET fusion in 24 "pan-negative" samples identified from the 202 lung adenocarcinomas from never smokers. Except for lung cancer sample 181LC, 261LC also harbors the CCDC6-RET fusion. LC: lung cancer. NL: normal lung.

shown), consistent with the mutual exclusive pattern of oncogenic drivers. Together with our recent identification of the CD74-ROS1 fusion in another "pan-negative" sample $[9,19]$, we have further improved our original work and uncovered the oncogenic drivers in about $94 \%$ (49/52) of these lung adenocarcinoma from never smokers [10]: EGFR mutations (78.8\%), HER2 mutations (3.8\%), KRAS mutations (1.9\%), EML4-ALK fusions (5.8\%), CD74-ROS1 fusion (1.9\%) and CCDC6-RET fusion $(1.9 \%)$ (Figure 1F).

Recently we have expanded the study of oncogenic mutation spectrum from the original 52 sample set to a large cohort with additional 150 samples and identified a total of 24 "pan-negative" lung adenocarcinomas from never smokers [19]. In an effort to detect the RET fusion in these "pan-negative" lung cancer samples, we found another lung cancer sample 261LC positive for CCDC6$R E T$ fusion (Figure $1 \mathrm{G}$ ). No other RET fusions including the recently reported KIF5B-RET fusion were found in these "pan-negative" samples [20] (data not shown). Interestingly, both patients with RET fusion are females; one is 46 years old with lung adenocarcinoma and the other is 61 years old with bronchioloalveolar carcinoma. Collectively, about $1 \%(2 / 202)$ of lung adenocarcinomas from never smokers harbors the CCDC6-RET fusion. Our data have not only provided a method for detection of novel oncogenic gene fusions but also constructed a more comprehensive map for oncogenic drivers in this subset of disease, which potentially helps develop the strategies for molecular-targeted therapies in clinic.

Experimental materials and methods are depicted in the Supplementary information, Data S1.

\section{Acknowledgments}

We thank Dr Bin Gao and Minghong He for technical supports. This work was supported by the National Basic Research Program of China (2010CB912102, 2012CB910800), Key Construction Program of the National "985" Project (985III-YFX 0102), the National Natural Science Foundation of China (30971461, 81101583,
81172218). H J is a scholar of the Hundred Talents Program of the Chinese Academy of Sciences and also gratefully acknowledges the support of SA-SIBS Scholarship Program.

Fei $\mathrm{Li}^{1,{ }^{*}}$, Yan Feng ${ }^{1, *}$, Rong Fang ${ }^{1, *}$, Zhaoyuan Fang ${ }^{1}$, Jufeng Xia ${ }^{1}$, Xiangkun Han ${ }^{1}$, Xin-Yuan Liu ${ }^{1}$, Haiquan Chen ${ }^{2,3}$, Hongyan Liu ${ }^{1}$, Hongbin $\mathrm{Ji}^{1}$

${ }^{I}$ State Key Laboratory of Cell Biology, Institute of Biochemistry and Cell Biology, Shanghai Institutes for Biological Sciences, Chinese Academy of Sciences, Shanghai 200031, China; ${ }^{2}$ Department of Thoracic Surgery, Fudan University Shanghai Cancer Center, Shanghai 200032, China; ${ }^{3}$ Department of Oncology, Shanghai Medical College, Fudan University, Shanghai 200032, China

*These three authors contributed equally to this work.

Correspondence: Hongbin $\mathrm{Ji}^{\mathrm{a}}$, Hongyan Liu ${ }^{\mathrm{b}}$, Haiquan $\mathrm{Chen}^{\mathrm{c}}$

a'E-mail: hbji@sibs.ac.cn

bE-mail: hyliudycui@yahoo.com.cn

cE-mail: hqchen1@yahoo.com

\section{References}

1 Toh CK, Gao F, Lim WT, et al. Never-smokers with lung cancer: epidemiologic evidence of a distinct disease entity. J Clin Oncol 2006; 24:2245-2251.

2 Subramanian J, Govindan R. Lung cancer in never smokers: a review. J Clin Oncol 2007; 25:561-570.

3 Herbst RS, Lippman SM. Molecular signatures of lung cancer-toward personalized therapy. N Engl J Med 2007; 356:76-78.

4 Lynch TJ, Bell DW, Sordella R, et al. Activating mutations in the epidermal growth factor receptor underlying responsiveness of non-small-cell lung cancer to gefitinib. $N$ Engl J Med 2004; 350:2129-2139.

5 Pao W, Miller VA. Epidermal growth factor receptor mutations, small-molecule kinase inhibitors, and non-small-cell lung cancer: current knowledge and future directions. J Clin Oncol 2005; 23:2556-2568.

6 Paez JG, Janne PA, Lee JC, et al. EGFR mutations in lung cancer: correlation with clinical response to gefitinib therapy. Science 2004; 304:1497-1500.

7 Koivunen JP, Mermel C, Zejnullahu K, et al. EML4-ALK fusion gene and efficacy of an ALK kinase inhibitor in lung can- 
cer. Clin Cancer Res 2008; 14:4275-4283.

8 Soda M, Choi YL, Enomoto M, et al. Identification of the transforming EML4-ALK fusion gene in non-small-cell lung cancer. Nature 2007; 448:561-566.

9 Sun Y, Ren Y, Fang Z, et al. Lung adenocarcinoma from East Asian never-smokers is a disease largely defined by targetable oncogenic mutant kinases. J Clin Oncol 2010; 28:4616-4620.

10 Rikova K, Guo A, Zeng Q, et al. Global survey of phosphotyrosine signaling identifies oncogenic kinases in lung cancer. Cell 2007; 131:1190-1203.

11 Wang L, Motoi T, Khanin R, et al. Identification of a novel, recurrent HEY1-NCOA2 fusion in mesenchymal chondrosarcoma based on a genome-wide screen of exon-level expression data. Genes Chromosomes Cancer 2012; 51:127-139.

12 Lin E, Li L, Guan Y, et al. Exon array profiling detects EML4ALK fusion in breast, colorectal, and non-small cell lung cancers. Mol Cancer Res 2009; 7:1466-1476.

13 Grieco M, Santoro M, Berlingieri MT, et al. PTC is a novel rearranged form of the ret proto-oncogene and is frequently detected in vivo in human thyroid papillary carcinomas. Cell 1990; 60:557-563.

14 Takahashi M. The GDNF/RET signaling pathway and human diseases. Cytokine Growth Factor Rev 2001; 12:361-373.
15 Fugazzola L, Pilotti S, Pinchera A, et al. Oncogenic rearrangements of the RET proto-oncogene in papillary thyroid carcinomas from children exposed to the Chernobyl nuclear accident. Cancer Res 1995; 55:5617-5620.

16 Santoro M, Melillo RM, Grieco M, et al. The TRK and RET tyrosine kinase oncogenes cooperate with ras in the neoplastic transformation of a rat thyroid epithelial cell line. Cell Growth Differ 1993; 4:77-84.

17 Jhiang SM, Sagartz JE, Tong Q, et al. Targeted expression of the ret/PTC1 oncogene induces papillary thyroid carcinomas. Endocrinology 1996; 137:375-378.

18 Santoro M, Chiappetta G, Cerrato A, et al. Development of thyroid papillary carcinomas secondary to tissue-specific expression of the RET/PTC1 oncogene in transgenic mice. Oncogene 1996; 12:1821-1826.

19 Li C, Fang R, Sun Y, et al. Spectrum of oncogenic driver mutations in lung adenocarcinomas from East Asian never smokers. PLoS One 2011; 6:e28204.

$20 \mathrm{Ju}$ YS, Lee WC, Shin JY, et al. A transforming KIF5B and RET gene fusion in lung adenocarcinoma revealed from whole-genome and transcriptome sequencing. Genome Res 2012; 22:436-445.

(Supplementary information is linked to the online version of the paper on the Cell Research website.) 